\title{
High-Density Lipoproteins-Associated Proteins and Subspecies Related to Arterial Stiffness in Young Adults with Type 2 Diabetes Mellitus
}

\author{
Xiaoting Zhu, ${ }^{1,2}$ Amy S. Shah, ${ }^{3}$ Debi K. Swertfeger, ${ }^{2}$ Hailong Li, ${ }^{4}$ Sheng Ren, \\ John T. Melchior, ${ }^{5}$ Scott M. Gordon, ${ }^{6}$ W. Sean Davidson, ${ }^{5}$ and L. Jason Lu $\mathbb{D}^{2}$ \\ ${ }^{1}$ School of Information Management, Wuhan University, Wuhan 430072, China \\ ${ }^{2}$ Division of Biomedical Informatics, Cincinnati Children's Hospital Research Foundation, 3333 Burnet Avenue, MLC 7024, \\ Cincinnati, OH 45229-3039, USA \\ ${ }^{3}$ Division of Endocrinology, Cincinnati Children's Hospital Research Foundation, 3333 Burnet Avenue, MLC 7012, Cincinnati, \\ OH 45229-3039, USA \\ ${ }^{4}$ Division of Neonatology and Pulmonary Biology, Cincinnati Children's Hospital Research Foundation, 3333 Burnet Avenue, \\ Cincinnati, OH 45229-3039, USA \\ ${ }^{5}$ Department of Pathology and Laboratory Medicine, Division of Experimental Pathology, University of Cincinnati, \\ 2120 East Galbraith Road, Cincinnati, OH 45237-0507, USA \\ ${ }^{6}$ Lipoprotein Metabolism Section, National Heart, Lung and Blood Institute, National Institutes of Health, Bethesda, MD, USA
}

Correspondence should be addressed to L. Jason Lu; long.lu@cchmc.org

Received 25 August 2017; Accepted 11 January 2018; Published 8 March 2018

Academic Editor: Mirjana Popović

Copyright (c) 2018 Xiaoting Zhu et al. This is an open access article distributed under the Creative Commons Attribution License, which permits unrestricted use, distribution, and reproduction in any medium, provided the original work is properly cited.

Lower plasma levels of high-density lipoproteins (HDL) in adolescents with type 2 diabetes (T2D) have been associated with a higher pulse wave velocity (PWV), a marker of arterial stiffness. Evidence suggests that HDL proteins or particle subspecies are altered in T2D and these may drive these relationships. In this work, we set out to reveal any specific proteins and subspecies that are related to arterial stiffness in youth with T2D from proteomics data. Plasma and PWV measurements were previously acquired from lean and T2D adolescents. Each plasma sample was separated into 18 fractions and evaluated by mass spectrometry. Then, we applied a validated network-based computational approach to reveal HDL subspecies associated with PWV. Among 68 detected phospholipid-associated proteins, we found that seven were negatively correlated with PWV, indicating that they may be atheroprotective. Conversely, nine proteins show positive correlation with PWV, suggesting that they may be related to arterial stiffness. Intriguingly, our results demonstrate that apoA-I and histidine-rich glycoprotein may reverse their protective roles and become antagonistic in the setting of T2D. Furthermore, we revealed two arterial stiffness-associated HDL subspecies, each of which contains multiple PWV-related proteins. Correlation and disease association analyses suggest that these HDL subspecies might link T2D to its cardiovascular-related complications.

\section{Background}

Type 2 diabetes mellitus (T2D) is a major risk factor for development of cardiovascular disease (CVD) [1]. An important contributor to elevating CVD risk in persons with T2D is dyslipidemia [2], characterized by high concentrations of small dense low-density lipoproteins (LDL) particles and serum triglycerides and low concentrations of high-density lipoprotein-cholesterol (HDL-C). Therapies focusing on improving CVD risk in T2D have largely focused on lowering atherogenic LDL particles. Unfortunately, even with the most effective LDL-lowering drugs, significant residual incidence of CVD remains in this population [3]. This has prompted numerous clinical trials aimed at raising HDL-C [4-6]. However, despite successfully raising HDL-C by $>70 \%$, these drugs failed to reduce CVD risk [4, 6, 7]. One recent exception was able to reduce CVD over statin treatment, but the effect was relatively modest [8]. 
HDL-targeted therapies have significant underlying challenges because $\mathrm{HDL}$ is more complex than previously recognized. Proteomic studies of HDL have identified at least 95 distinct proteins associated with HDL [9-14]. It is impossible for all of these proteins to reside on a single particle. Instead, it is likely that several proteins interact on different particles, likely to carry out specific functions. A perfect example is trypanosome lytic factor (TLF), containing apolipoprotein A-I (apoA-I), haptoglobin-related protein, and apoL-I. This particle has been shown to have specific lytic activity against the protozoan Trypanosoma brucei via lysosomal disruption [15].

Recent studies have shown that HDL is a heterogeneous group of subspecies with unique protein/lipid compositions that are not reflected in the HDL-C measurement [13, 16-18]. Diverse proteins on HDL subspecies may have physical interactions or be colocalized on the same particle without a physical interaction. Those different HDL subspecies provide a plausible explanation for the many diverse functions of HDL [19]. While HDL is most recognized for its ability to mediate reverse cholesterol transport (RCT) [20], in which it removes excessive cholesterol and other lipids from peripheral tissues and transports them back to the liver for catabolism, HDL has also been shown to have anti-inflammatory, antioxidative, and antiapoptotic properties [21-25].

Using gel filtration chromatography that separates lipoproteins by size, we previously found that adolescents with type 2 diabetes were depleted of large HDL particles compared with obese and lean adolescents [17]. This decrease was inversely and significantly correlated with an increase in pulse wave velocity (PWV), an early measurement of arterial stiffness. Additionally, we detected $45 \mathrm{HDL}$-associated proteins, seven of which were lower in the T2D group. These results demonstrated an alteration in the HDL composition in young adults with T2D. However, in our previous paper, we had not examined if specific proteins or protein subspecies on these particles may be related to arterial stiffness.

Here we sought to determine if any proteins or groups of proteins may be related to arterial stiffness as measured by PWV. Recently, we have developed a network-based computational method to infer HDL subspecies from complex proteomics data [16]. Supported by multiple lines of evidence, the protein clusters revealed by our method likely correspond to HDL subspecies. In this work, we integrated patient clinical data, biological experiments, and bioinformatics analysis to reveal arterial stiffness-related HDL proteins and subspecies, which may serve as better markers than HDL-C for linking T2D with CVD.

\section{Methods}

2.1. Study Cohort. Five adolescents with $\mathrm{T} 2 \mathrm{D}$ and six lean controls aged 17-27 years were included in this analysis [17]. Table 1 lists the clinical characteristics of the study cohort. Subjects with T2D were diagnosed based on American Diabetes Association criteria [26]. The lean control group had a BMI less than the 85th percentile (age $\leq 20$ ) or less than 25 (age $>20$ ). Lean control participants had no evidence of chronic disease. Manual blood pressure was measured three times and averaged using a mercury sphygmomanometer. Arterial stiffness was measured by carotid to femoral PWV, a risk factor for coronary artery disease and stroke [27]. For each subject, PWV was collected using a SphygmoCor SCORPVx System. Three PWV values for each participant were measured and averaged.

2.2. Plasma Fractionation and Proteomics. For each subject, blood was fractionated by gel filtration chromatography into 18 lipid-containing fractions by size [13]. By this method, which has been extensively described $[13,18]$, fractions 13-30 contain detectable phospholipid that corresponds to the plasma lipoproteins VLDL, LDL, and HDL. To relate gel filtration results to traditional density-centric definitions, the VLDL/LDL range was defined as fractions 14-18 due to the presence of apoB. The remaining fractions 19-30 were defined as a HDL range because their diameters are consistent with measurements for density-isolated HDL and because of the abundance of the major HDL protein, apoAI. Lipid-associated proteins were isolated using a synthetic calcium silicate hydrate, Lipid Removal Agent (Supelco), and their relative abundance in each fraction was determined by mass spectrometry (MS) as previously described [17, 28]. Briefly, samples were delipidated using chloroform and methanol. Next, samples were treated with dithiothreitol and iodoacetamide (Sigma-Aldrich, St. Louis, MO) to reduce and carbamidomethylate the proteins. Finally, proteins were trypsinized and subjected to MS. An Agilent 1100 Series Autosampler/HPLC was used to draw $0.5 \mu \mathrm{L}$ of sample and inject it onto a C18 reverse phase column (GRACE; 150 $\times 0.500 \mathrm{~mm}$ ), where an acetonitrile concentration gradient (5-30\% in water with $0.1 \%$ formic acid) was used to elute peptides for inline electrospray ionization tandem MS, by a time of flight QSTAR XL mass spectrometer (Applied Biosystems). Column cleaning was performed automatically with two cycles of a 5-85\% acetonitrile gradient lasting 15 min each between runs. PeakView version 2.1 was used to convert the raw data files into the peak list (.mgf) files. The resulting mass spectra were analyzed with Mascot (version 2.2.2, http://www.matrixscience.com) and X! Tandem (version 2001.01.01.1) search engines against the UniProtKB/Swiss-Prot Protein Knowledgebase (2011, containing 540,958 entries). Search criteria included human taxonomy and carbamidomethylation as a variable modification; peptide tolerance was set at $\pm 20 \mathrm{ppm}, \mathrm{MS} / \mathrm{MS}$ tolerance was set to $\pm 0.6 \mathrm{Da}$, and up to 3 missed trypsin cleavages were allowed. Peptide and protein identification from the MS/MS was validated using Scaffold software (version 3.3.1) and only peptides with $>95 \%$ identification probability and proteins with $>99 \%$ identification probability were included in the analysis. Additionally, at least 2 peptides from each protein were required to be considered in the analysis. False discovery rates were less than $0.6 \%$ for peptide identification (calculated as the percentage of the sum of exclusive spectrum counts of decoy proteins divided by the sum of exclusive spectrum counts of target proteins) and less than $0.1 \%$ for protein identification (calculated as number of decoy proteins divided by the number of target proteins). MS peptide counts were obtained to semiquantitatively identify differences in 
TABLE 1: Study cohort characteristics.

\begin{tabular}{lcc}
\hline Variable & Lean & T2D \\
\hline$n$ & 6 & 5 \\
Age (years) & $21.67 \pm 2.16$ & $21.6 \pm 4.04$ \\
Body mass index (mg/kg) & $23.97 \pm 2.04$ & $37.89 \pm 3.75$ \\
Total cholesterol (mg/dL) & $153.67 \pm 26.38$ & $215.6 \pm 55.84$ \\
Triglycerides (mg/dL) & $73.17 \pm 27.8$ & $278.6 \pm 344.52$ \\
High-density lipoprotein-cholesterol (mg/dL) & $52.67 \pm 10.73$ & $33.8 \pm 4.44$ \\
Low-density lipoprotein-cholesterol (mg/dL) & $86.6 \pm 32.24$ & $127.8 \pm 53.1$ \\
Systolic blood pressure (mmHg) & $119.72 \pm 11.33$ & $116.0 \pm 8.21$ \\
Diastolic blood pressure (mmHg) & $71.56 \pm 7.96$ & $71.2 \pm 5.78$ \\
Pulse wave velocity (m/s) & $5.74 \pm 1.11$ & $6.8 \pm 1.12$ \\
Hemoglobin AlC $(\%)$ & N/A & $11.3 \pm 4.65$ \\
Hemoglobin AlC (mmol/mol) & N/A & $100.2 \pm 50.63$ \\
\hline
\end{tabular}

Data are mean and standard deviation (SD). N/A: not available.

proteins across fractions between groups. Although peptide spectral counting has been criticized for its accuracy to reflect the abundance of proteins, it would not compromise our analysis in this work, since we only considered the trends or distribution patterns of HDL proteins. Spectral counts were used in no instance to reflect the absolute quantity of a protein. For data preprocessing, we filtered the MS detected proteins with the HDL Proteome Watch [29] in all lean and T2D participants. The HDL Proteome Watch is a curated database documenting all proteins that have been found to be associated with HDL in multiple MS studies. We further removed extremely low-abundant proteins (less than 3 peptides across all subjects).

2.3. Correlation Analysis. To investigate if any individual HDL proteins were related to arterial stiffness, we calculated Pearson correlation coefficient (PCC) between the relative abundance of HDL proteins in fractions 19-31 and PWV measurements. PCC was calculated for the lean and T2D combined dataset, the lean dataset only, and T2D dataset only. We realized that PCC of the lean and T2D combined dataset may be mainly driven by the lean group (Supplemental Figure 1). If the composition of HDL is indeed altered in T2D, it is possible that, in the same size fractions, lean and T2D datasets exhibit distinct patterns. Thus, in this work, we separated lean and T2D groups and analyzed them individually. Specifically, for each plasma HDL fraction $\epsilon\left[\begin{array}{lll}19 & \cdots & 30\end{array}\right]$, we calculated a univariate PCC between PWV and the peptide counts of HDL-associated proteins in fractions across all subjects within the two groups. We applied a two-tailed $t$-test followed by the Benjamini-Hochberg procedure to correct for multiple comparisons to test the null hypothesis of no correlation between PWV and proteins.

2.4. Network-Based Identification of HDL Subspecies. To infer HDL subspecies, we applied the network-based approach we previously developed for clustering comigrating proteins [16]. This approach is based on the assumption that proteins with similar elution patterns during plasma fractionation are very likely to reside on the same HDL subspecies. We constructed a comigration protein-protein interaction (PPI) network for the lean and T2D groups, separately, using fractions 13-30. Comigration similarity was measured by univariate PCC, and strongly correlated links are chosen to form the networks. Proteins in the network were clustered using a network clustering approach, ClusterONE [30]. The identified protein clusters found in fractions 19-30 were inferred to be HDL subspecies.

2.5. Disease Association Analysis. To determine whether the HDL subspecies are related to particular diseases, we performed disease association analyses using an independent analyzer, ToppCluster [31]. In disease association analysis, ToppCluster calculated an enrichment score with a hypergeometric distribution test for our identified HDL subspecies associated with human disease annotations that were collected from multiple public human disease databases. A default Bonferroni correction was applied on the tests of associations and the corrected $P<0.05$ was used to select significant associated diseases.

\section{Results}

3.1. HDL-Associated Proteins and Arterial Stiffness. We first sought to determine if individual HDL-associated proteins in the plasma HDL fractions were associated with arterial stiffness. A total of $68 \mathrm{HDL}$-associated proteins were identified across all fractions. Figures 1 and 2 show univariate PCC of 58 proteins within the HDL size fractions versus PWV in the lean and T2D groups, separately. Across the two groups, we identified 14 distinct HDL proteins that exhibited significant correlation with $\mathrm{PWV}(|\mathrm{PCC}| \geq 0.9, P<0.05$ after Benjamini-Hochberg procedure). In the lean group specifically, seven proteins showed strong negative correlation with PWV (Figure 1). Those include classical apolipoproteins apoA-I, apoC-III, and apoJ, as well as complement Cls subcomponent, histidine-rich glycoprotein, inter-alpha-trypsin inhibitor 2, and pigment epithelium-derived factor. The significant proteins were detected across the HDL size range and showed no preferential distribution in larger or smaller 


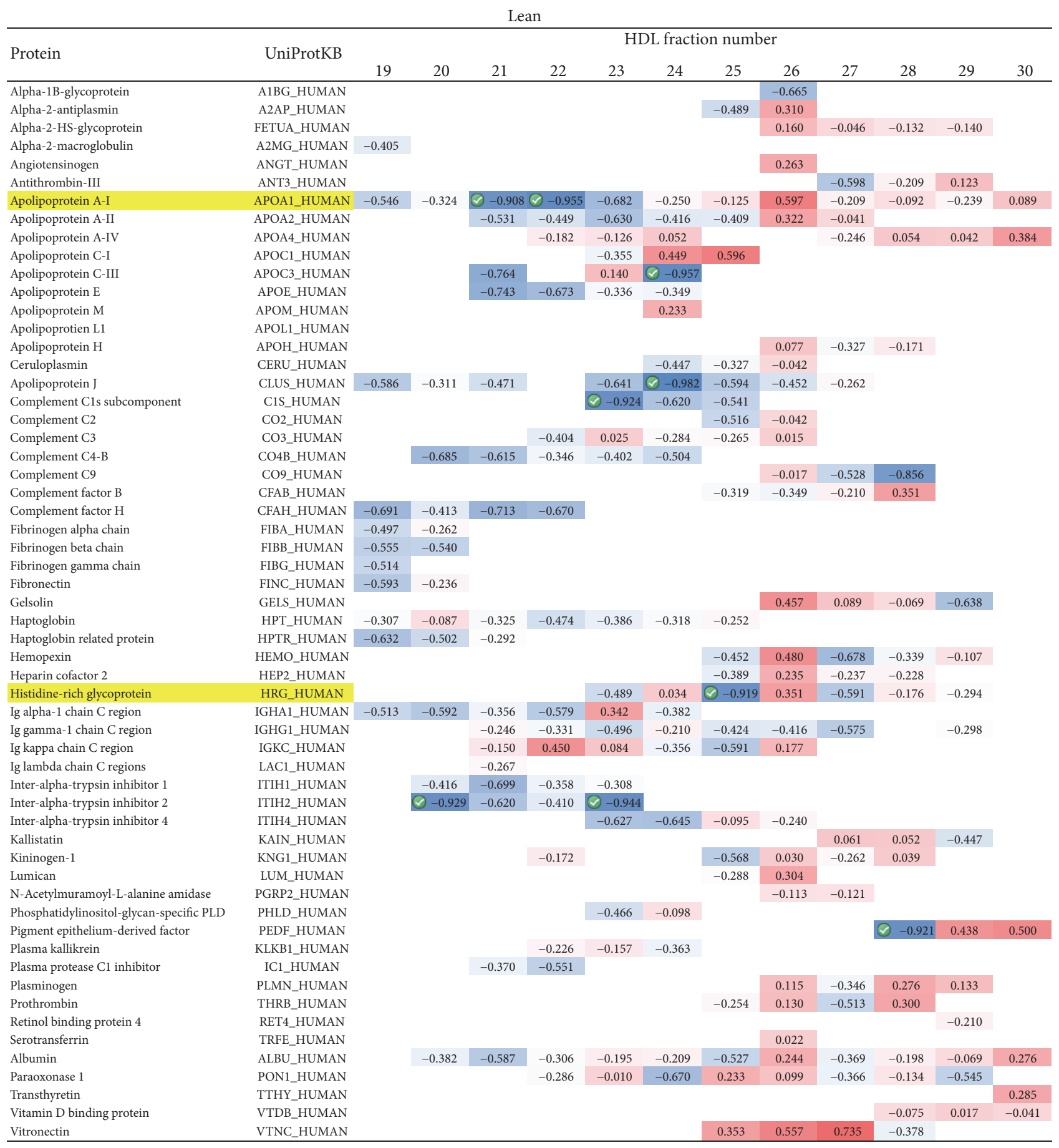

FIGURE 1: PCCs of peptide counts of individual proteins with PWV for the lean group across HDL fractions. Red, white, and blue are used to mark maximal, medium, and minimum value, respectively. Green checkmark indicates significant correlation. The proteins that also correlate with PWV in the T2D group are highlighted in yellow.

particles. The negative correlation with PWV suggests that these proteins may be components of the atheroprotective particles.

In contrast, in the $\mathrm{T} 2 \mathrm{D}$ group, nine proteins were found to be positively correlated with PWV (Figure 2). These proteins were mainly distributed across fractions 26 29, the range containing smaller HDL particles. These proteins were
alpha-2-HS-glycoprotein, antithrombin-III, apoA-I, gelsolin, hemopexin, histidine-rich glycoprotein, kininogen-1, plasminogen, and albumin. The positive correlation with PWV suggests that these may be atherogenic. It is worth noting that apoA-I and histidine-rich glycoprotein were found in both groups but with negative correlation with PWV in the lean group and positive correlation in the T2D group, 
$\mathrm{T} 2 \mathrm{D}$

\begin{tabular}{|c|c|c|c|c|c|c|c|c|c|c|c|c|c|}
\hline \multirow{2}{*}{ Protein } & \multirow{2}{*}{ UniProtKB ID } & \multicolumn{12}{|c|}{ HDL fraction number } \\
\hline & & 19 & 20 & 21 & 22 & 23 & 24 & 25 & 26 & 27 & 28 & 29 & 30 \\
\hline Alpha-1B-glycoprotein & A1BG_HUMAN & & & & & & & & 0.767 & & & & \\
\hline Alpha-2-antiplasmin & A2AP_HUMAN & & & & & & & & & & & & \\
\hline Alpha-2-HS-glycoprotein & FETUA_HUMAN & & & & & & & & (6) 0.934 & 0.838 & 0.577 & & \\
\hline Alpha-2-macroglobulin & A2MG_HUMAN & & & & & & & & & & & & \\
\hline Angiotensinogen & ANGT_HUMAN & & & & & & & & & & & & \\
\hline Antithrombin-III & ANT3_HUMAN & & & & & & & & & 0.669 & 0.864 & ( 0.905 & \\
\hline Apolipoprotein A-II & APOA2_HUMAN & & & & & & 0.000 & 0.320 & 0.848 & 0.442 & & & \\
\hline Apolipoprotein A-IV & APOA4_HUMAN & & & & & 0.842 & 0.230 & & & 0.842 & 0.703 & 0.550 & 0.381 \\
\hline Apolipoprotein C-I & APOC1_HUMAN & & & & & & & & & & & & \\
\hline Apolipoprotein C-III & APOC3_HUMAN & & & & & & & & & & & & \\
\hline Apolipoprotein E & APOE_HUMAN & & & & & 0.856 & & & & & & & \\
\hline Apolipoprotein $\mathrm{M}$ & APOM_HUMAN & & & & & & & & & & & & \\
\hline Apolipoprotien L1 & APOL1_HUMAN & & -0.189 & & & & & & & & & & \\
\hline Apolipoprotein $\mathrm{H}$ & APOH_HUMAN & & & & & & & & 0.347 & 0.764 & 0.861 & & \\
\hline Complement $\mathrm{C} 1 \mathrm{~s}$ subcomponent & C1S_HUMAN & & & & & & 0.269 & & & & & & \\
\hline Complement $\mathrm{C} 2$ & $\mathrm{CO} 2$ _HUMAN & & & & & & & -0.100 & & & & & \\
\hline Complement C3 & CO3_HUMAN & & & & 0.708 & 0.320 & 0.282 & 0.719 & & & & & \\
\hline Complement C4-B & CO4B_HUMAN & & & & 0.417 & 0.328 & -0.024 & & & & & & \\
\hline Complement $\mathrm{C} 9$ & CO9_HUMAN & & & & & & & & -0.491 & 0.848 & -0.191 & & \\
\hline Complement factor B & CFAB_HUMAN & & & & & & & 0.257 & 0.795 & 0.562 & 0.698 & & \\
\hline Complement factor $\mathrm{H}$ & CFAH_HUMAN & 0.587 & 0.898 & 0.382 & & & & & & & & & \\
\hline Fibrinogen alpha chain & FIBA_HUMAN & 0.364 & & & & & & & & & & & \\
\hline Fibrinogen beta chain & FIBB_HUMAN & 0.366 & & & & & & & & & & & \\
\hline Fibrinogen gamma chain & FIBG_HUMAN & 0.097 & & & & & & & & & & & \\
\hline Fibronectin & FINC_HUMAN & 0.736 & 0.342 & & & & & & & & & & \\
\hline Gelsolin & GELS_HUMAN & & & & & & & & 0.848 & (6) 0.921 & 0.804 & & \\
\hline Haptoglobin & HPT_HUMAN & -0.158 & 0.323 & 0.122 & 0.471 & 0.442 & -0.006 & -0.031 & & & & & \\
\hline Haptoglobin related protein & HPTR_HUMAN & 0.424 & -0.052 & -0.200 & & & & & & & & & \\
\hline Hemopexin & HEMO_HUMAN & & & & & & & 0.827 & 0.883 & 0.751 & ( 0.904 & & \\
\hline Heparin cofactor 2 & HEP2_HUMAN & & & & & & & -0.021 & 0.632 & 0.684 & 0.366 & & \\
\hline Histidine-rich glycoprotein & HRG_HUMAN & & & & & & & 0.736 & 0.890 & 0.786 & 0.959 & & \\
\hline Ig kappa chain $\mathrm{C}$ region & IGKC_HUMAN & & & & & 0.316 & & & & & & & \\
\hline Ig lambda chain $\mathrm{C}$ regions & LAC1_HUMAN & & & & & & & & & & & & \\
\hline Inter-alpha-trypsin inhibitor 1 & ITIH1_HUMAN & & 0.736 & 0.496 & 0.590 & & & & & & & & \\
\hline Inter-alpha-trypsin inhibitor 2 & ITIH2_HUMAN & & 0.424 & 0.000 & 0.258 & & & & & & & & \\
\hline Inter-alpha-trypsin inhibitor 4 & ITIH4_HUMAN & & & & & & 0.426 & 0.414 & & & & & \\
\hline Kallistatin & KAIN_HUMAN & & & & & & & & & & 0.817 & 0.574 & \\
\hline Kininogen-1 & KNG1_HUMAN & & & & & & & 0.267 & 0.632 & 0.947 & 0.574 & & \\
\hline Lumican & LUM_HUMAN & & & & & & & & & & & & \\
\hline $\mathrm{N}$-Acetylmuramoyl-L-alanine amidase & PGRP2_HUMAN & & & & & & & & 0.126 & -0.133 & & & \\
\hline Phosphatidylinositol-glycan-specific PLD & PHLD_HUMAN & & & & & & & & & & & & \\
\hline Pigment epithelium-derived factor & PEDF_HUMAN & & & & & & & & & & 0.842 & 0.480 & 0.842 \\
\hline Plasma kallikrein & KLKB1_HUMAN & & & & 0.530 & 0.450 & 0.274 & & & & & & \\
\hline Plasma protease $\mathrm{C} 1$ inhibitor & IC1_HUMAN & & & & & & & & & & & & \\
\hline Plasminogen & PLMN_HUMAN & & & & & & & & 0.978 & 0.892 & 0.578 & & \\
\hline Prothrombin & THRB_HUMAN & & & & & & & 0.856 & 0.437 & 0.050 & & & \\
\hline Retinol binding protein 4 & RET4_HUMAN & & & & & & & & & & & & \\
\hline Serotransferrin & TRFE_HUMAN & & & & & & & & & & & & \\
\hline Albumin & ALBU_HUMAN & & & -0.126 & 0.319 & & 0.424 & & 0.670 & 0.900 & 0.870 & 0.782 & 0.869 \\
\hline Paraoxonase 1 & PON1_HUMAN & & & & & & & -0.126 & & & & & \\
\hline Transthyretin & TTHY_HUMAN & & & & & & & & & & & & \\
\hline Vitamin $\mathrm{D}$ binding protein & VTDB_HUMAN & & & & & & & & & & & 0.201 & \\
\hline Vitronectin & VTNC_HUMAN & & & & & & & 0.692 & 0.510 & 0.710 & 0.872 & & \\
\hline
\end{tabular}

FIGURE 2: PCCs of peptide counts of individual proteins with PWV for the T2D group across HDL fractions. Red, white, and blue are used to mark maximal, medium, and minimum value, respectively. Green checkmark indicates significant correlation. The proteins that also correlate with PWV in the lean group are highlighted in yellow.

suggesting that perhaps the protein distribution (what size particles it associates with) is altered in disease, rendering it proatherogenic. Except the significantly PWV-correlated proteins mentioned above, the majority of phospholipidassociated proteins demonstrated no clear correlation with PWV measurements.
3.2. Pattern Distribution of Proteins Associated with PWV. Based on the correlation analysis, we wanted to know if these PWV-related proteins are associated with altered HDL particle sizes. This prompted us to investigate the distributions of these PWV-related proteins. We compared distribution patterns of all the significantly correlated proteins between 


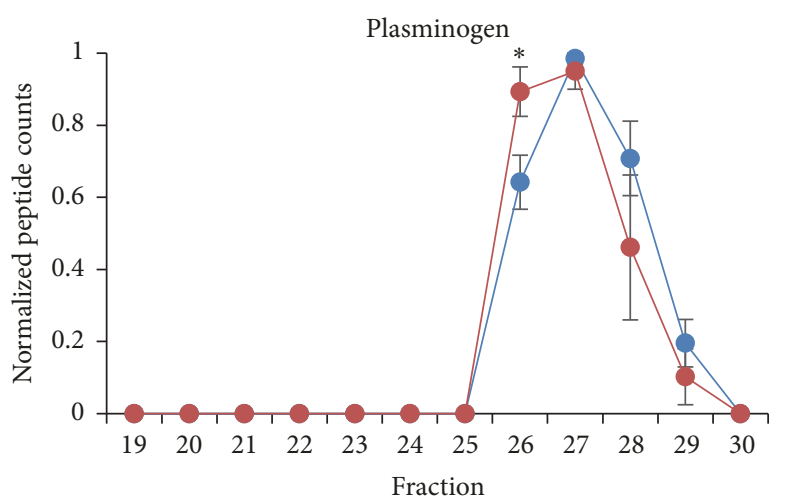

(a)

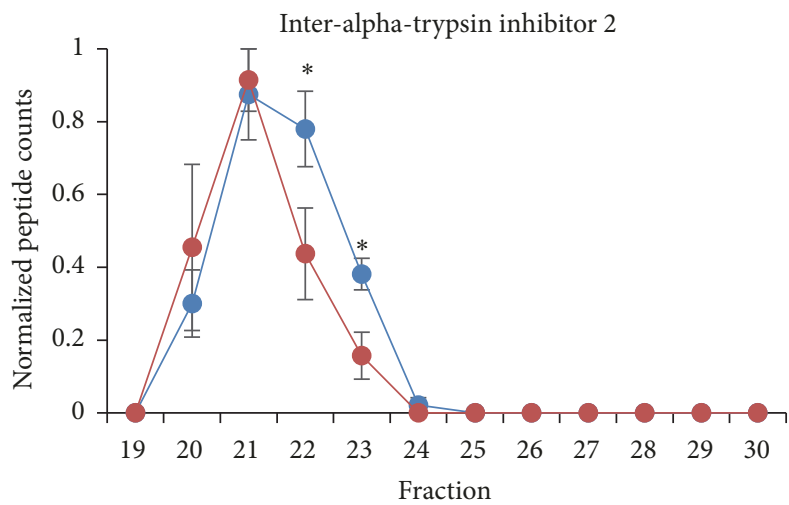

(c)

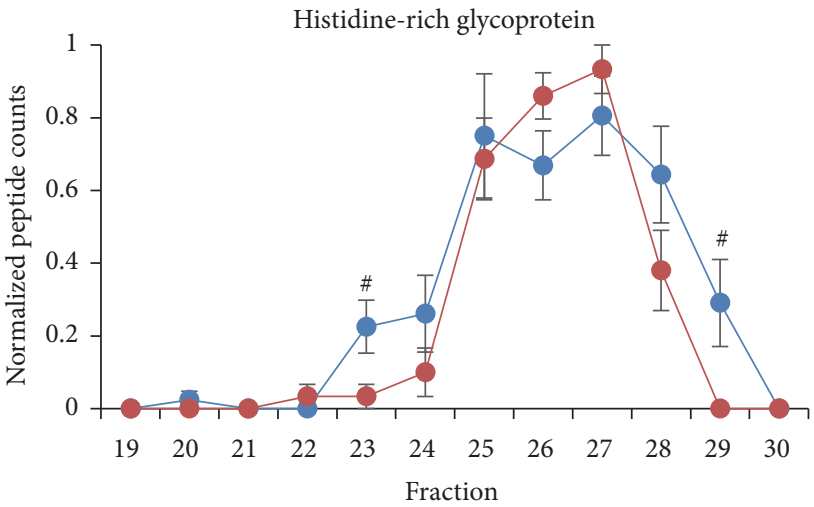

(e)

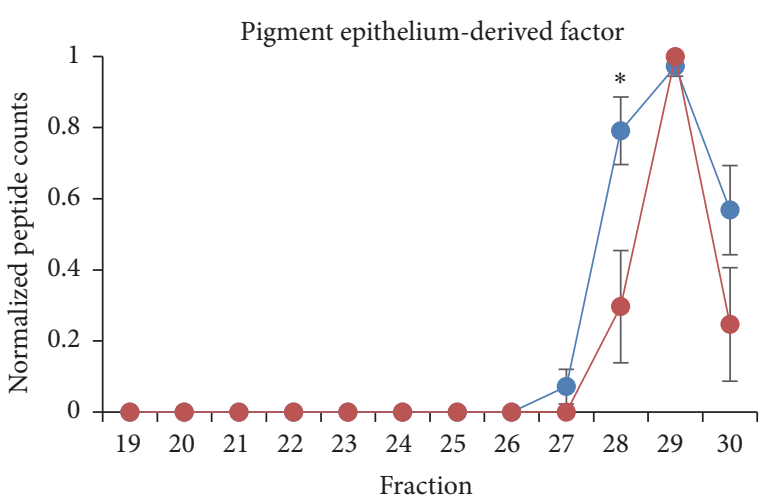

(b)

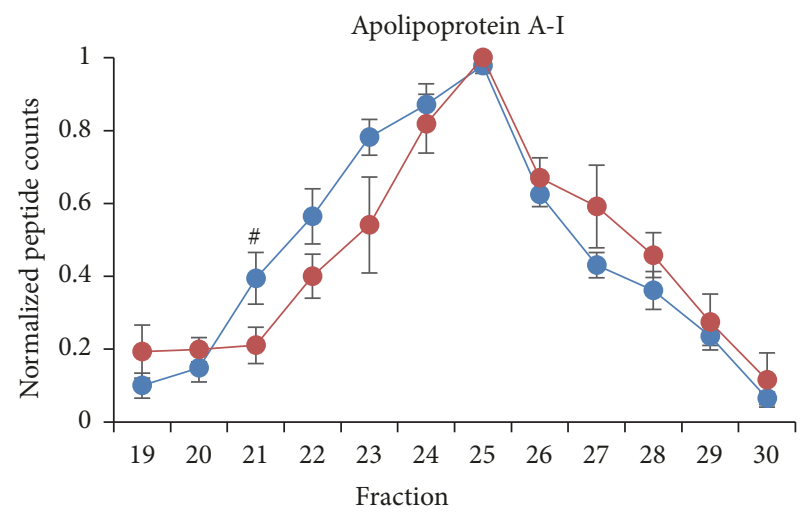

(d)

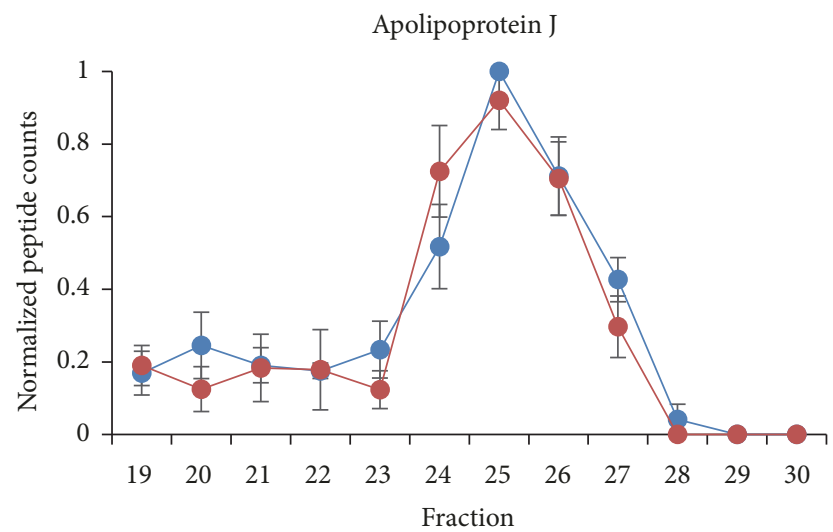

(f)

FIGURE 3: Distribution patterns of example proteins exhibiting high correlations with $P W V$. Spectral counts were normalized by dividing by the maximum value across all fractions. Data are mean and standard deviation of normalized spectral counts. Normalized peptide counts across the HDL fractions are shown for lean (blue) and T2D (red): (a) plasminogen, (b) pigment epithelium-derived factor, (c) inter-alphatrypsin inhibitor 2, (d) apoA-I, (e) histidine-rich glycoprotein, and (f) apoJ. A $t$-test was performed for comparing normalized peptide counts between lean and T2D groups at individual fractions. ${ }^{*} P<0.05 .{ }^{\#} P<0.08$.

the lean and T2D groups $(n=14)$. Using a $t$-test, we determined that 5/14 PWV-correlated proteins have significant distribution changes at specific fractions $(P<0.05)$. These include gelsolin, kininogen-1, plasminogen, inter-alphatrypsin inhibitor 2 (ITIH2), and pigment epithelium-derived factor (PEDF). We noted that an additional five proteins (i.e., apoA-I, alpha-2-HS-glycoprotein, hemopexin, histidine-rich glycoprotein, and albumin) exhibited observable pattern changes but their $P$ value was slightly greater than 0.05 .
Figures 3(a)-3(c) demonstrate PWV-correlated proteins with significant pattern changes. Plasminogen and PEDF were altered in smaller size HDL range, while ITIH2 was changed in the larger HDL size fractions. Figures 3(d) and 3(e) show two examples whose pattern changes are observable but not significant at certain fractions. For example, compared with the lean subjects, apoA-I is decreased in the T2D subjects at fraction 21 (Figure 3(d)) as well as histidine-rich glycoprotein at fraction 23 (Figure 3(e)). Their changes both appeared in 
the larger HDL size fractions. Only four of $14 \mathrm{PWV}$-correlated proteins exhibited no observable changes in distribution: antithrombin-III, apoC-III, complement Cls subcomponent, and apoJ (Figure 3(f)). The pattern analysis suggests that only a portion of HDL subspecies where these proteins reside were altered in youth with T2D.

3.3. HDL Subspecies and Arterial Stiffness. Next, we sought to identify potential HDL subspecies that may be related to arterial stiffness. We have previously designed a computational method to reveal putative HDL subspecies [16]. In this work, we employed the same approach to construct comigration PPI networks for the lean and T2D subjects, respectively, as described in Section 2. The spectral counts of the 68 proteins across fractions 13-30 were applied in network construction. PPI networks for the lean and T2D groups are shown in Figure 4. The networks only include strongly correlated comigration links ( $\mathrm{PCC} \geq 0.8, P<0.05)$ and, as a result, 42 associated HDL proteins. The lean network shown in Figure 4(a) contains 62 comigration links, while the T2D network in Figure 4(b) has only 35 links. Fibrinogen alpha chain, fibrinogen beta chain, and fibrinogen gamma chain (yellow) form fibrinogen, a glycoprotein in plasma. These three subunits should be interconnected in the comigration network, since they colocalize and travel together in fractionated plasma as a single protein. Interconnection of the three subunits in both Figures 4(a) and 4(b) was observed, validating our network construction.

In Figure 4(a), we identified several groups of proteins that can be found clustered together using the ClusterONE method [30]. These clusters may comprise distinct HDL subspecies and are marked with various colors in the lean controls. Nodes that are not in any cluster are marked with aqua color. In T2D network (Figure 4(b)), we applied the same layout and color scheme as in Figure 4(a) for comparison. There are global topological differences between the lean and T2D networks. A clustering coefficient (CC) was calculated to measure the degree to which nodes in a network tend to cluster together. The CC of the lean network is 0.508, while the CC of T2D network is reduced to 0.225 . This reduction indicates that lean subjects may have more intact protein subspecies than T2D subjects.

Another important topological feature of a network is the number of connected components (NCC). In a graph, its connected components are the set of the largest subgraphs that are each connected. A lower NCC indicates fewer subgraphs and suggests stronger network connectivity. The NCC of lean and T2D networks are 8 and 23, respectively. In terms of number of links, the lean network has more links than the T2D network with the same PCC cutoff. This suggests that HDL proteins in the lean controls have a stronger comigration relationship than the ones in T2D subjects. Loss of the links in the T2D network is mainly within the clusters (e.g., green and yellow clusters). On the other hand, the T2D network involves additional links; for example, kininogen-1 comigrates with the pink cluster. The differences between the networks indicate that the compositional alterations of certain HDL subspecies may occur in youth with T2D.
Upon further examination, we first noted that the edge between apoA-I and apoA-II is absent in the T2D group. Although not all apoA-I containing particles include apoAII, it is well known that apoA-I and apoA-II often reside on the same HDL complexes [14, 18, 29, 32]. An independent study [32] has reported the apoA-I and apoA-II comigrated after gel filtration-based plasma fractionation $(\mathrm{PCC}=0.96)$. In the current study, the PCC of the apoA-I and apoA-II migration pattern in the lean group is $0.871(P<0.05)$, while the PCC in T2D group is reduced to $0.747(P<0.05)$. Their comigration patterns were compared between lean and T2D groups (Figures 5(a) and 5(b)). Obvious distribution changes appeared on apoA-II, especially around fractions 21-23. This was previously reported in our original paper [17]. The lower PCC may be due to either a loss of the small HDL particle in T2D patients or the compositional alteration of HDL subspecies containing apoA-II in youth with T2D. Additionally, the link between haptoglobin-related protein and complement factor $\mathrm{H}$ is lost in the T2D network. Their comigration patterns (Figures 5(c) and 5(d)) demonstrate that haptoglobin-related protein has a clear pattern alteration in the T2D group. On the other hand, we found some edges that only appear in the T2D network.

Figures 5(e) and 5(f) show that kininogen-1 has a peak at fraction 26 in the lean controls but a peak at fraction 27 in the T2D subjects. This right-shift makes kininogen-1 have a similar migration pattern to apoH in youth with T2D.

In the HDL-associated PPI network, we observed three clusters that contain at least two proteins that were highly correlated with PWV. These are the blue cluster containing four proteins (apoA-IV, antithrombin-III, kallistatin, and albumin), the green cluster containing five proteins (apoAI, apoA-II, complement C3, complement C1s subcomponent, and Ig gamma-1 chain $\mathrm{C}$ region), and the pink cluster containing six proteins (hemopexin, gelsolin, plasminogen, complement C9, apoH, and alpha-2-HS-glycoprotein). The proteins in the blue cluster do not likely form a subspecies. They simply migrate together due to their similar sizes. If they formed a subspecies, their combined MW would be at least $205 \mathrm{kDa}$, without any associated lipid, and would thus elute in an earlier fraction. Therefore, this grouping will not be referred to as a subspecies. However, the remaining 2 clusters are each likely migrating together as at a subspecies, since they elute in a fraction containing proteins that are larger than any single protein in their respective clusters. The migration patterns for the 2 subspecies in both lean and T2D groups are shown in Figure 6. Again, we first provided fibrinogen as a control shown in Figures 6(a) and 6(b) to illustrate how different proteins comigrate if they reside on the same "particle" and show no difference in the pattern between lean and T2D as previously observed. The rest of panels in Figure 6 demonstrate the patterns of the two newly revealed clusters in lean and T2D. The green cluster (Figures 6(c) and 6(d)) contains two negatively PWV-correlated proteins that were identified in the lean group. Pattern changes are clear in the T2D group, so that links between proteins in the lean group are absent in the T2D group.

It appears that the intact subspecies containing two atheroprotective proteins are remodeled into disconnected 


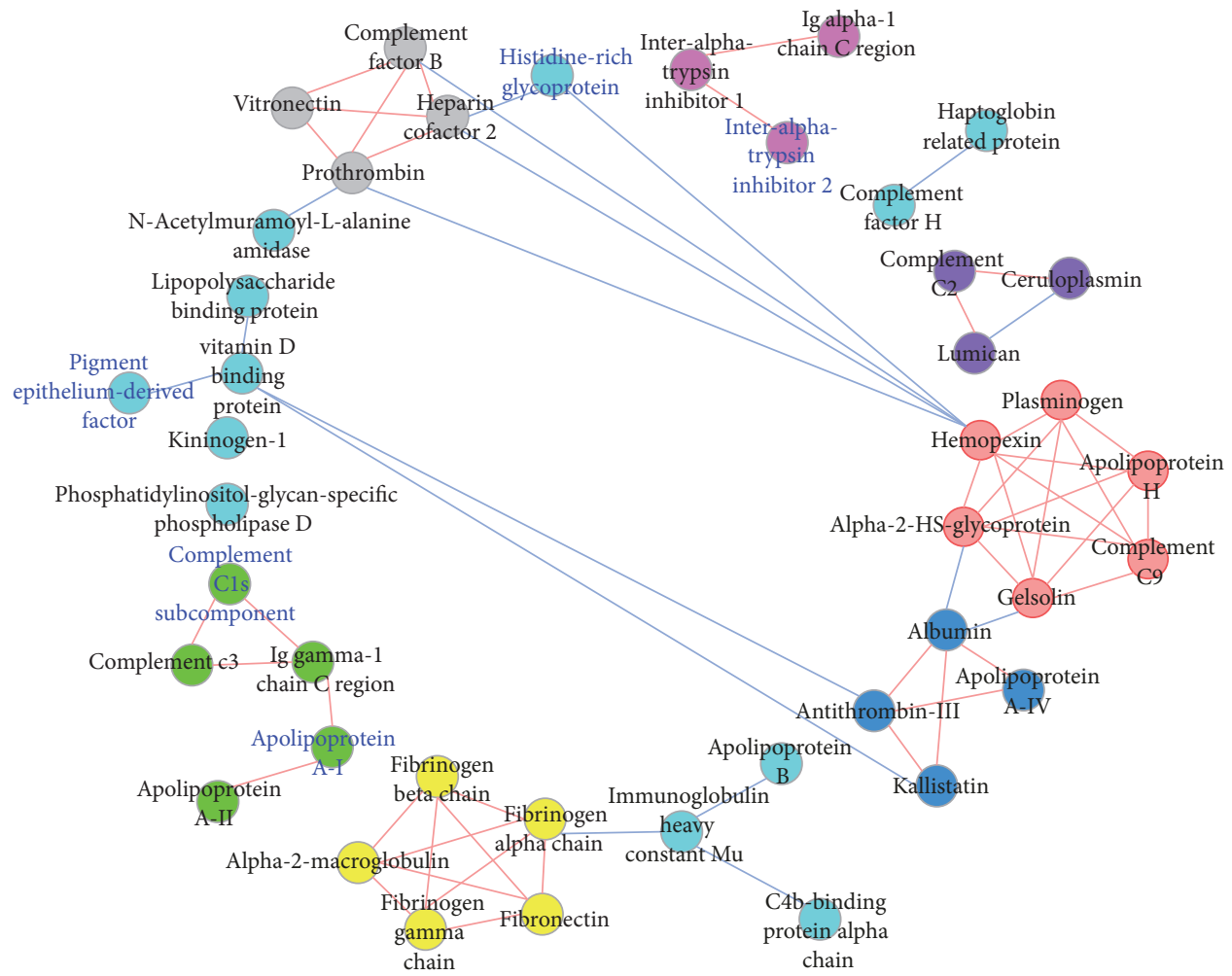

(a)

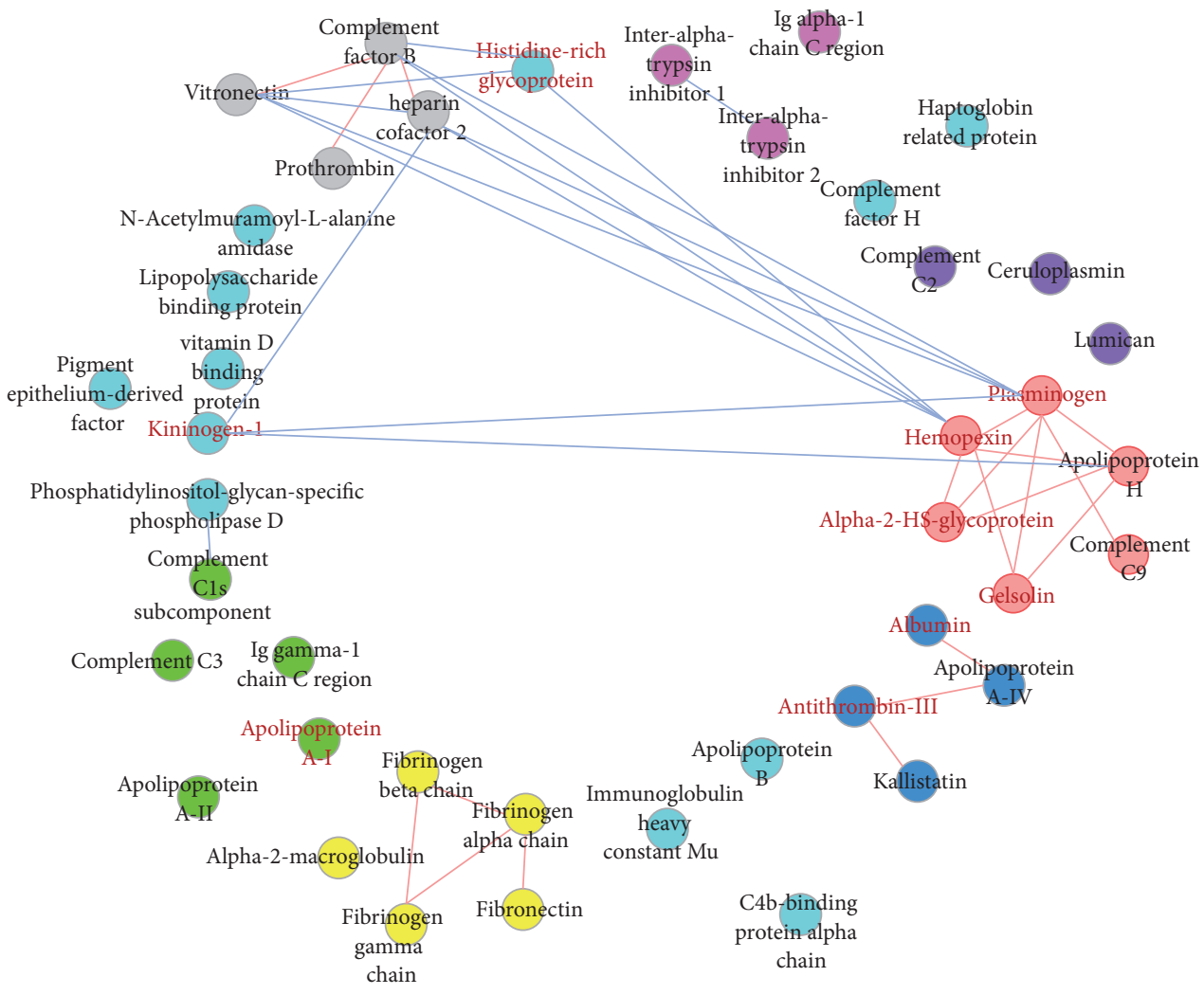

(b)

FIGURE 4: HDL-associated PPI networks for the (a) lean group and (b) T2D group. Nodes represent the proteins and edges represent comigration relationship. Intracluster edges are marked as dark pink, and intercluster edges are blue. Significantly PWV-correlated proteins are denoted with red text (positive) and blue text (negative). Clustered protein groups are marked with different colors. 


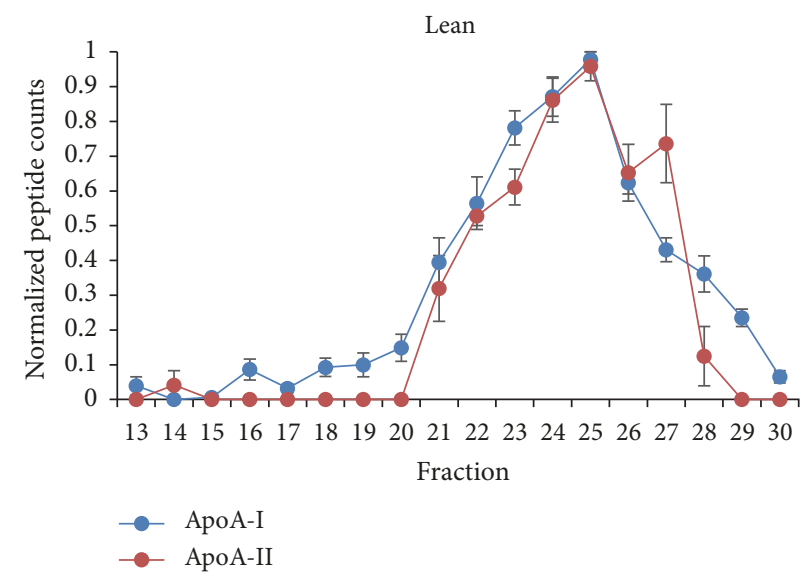

(a)

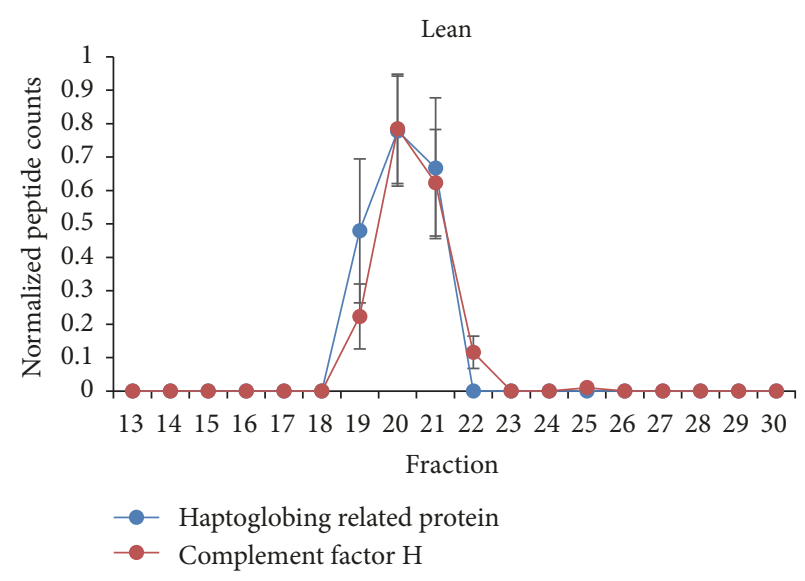

(c)

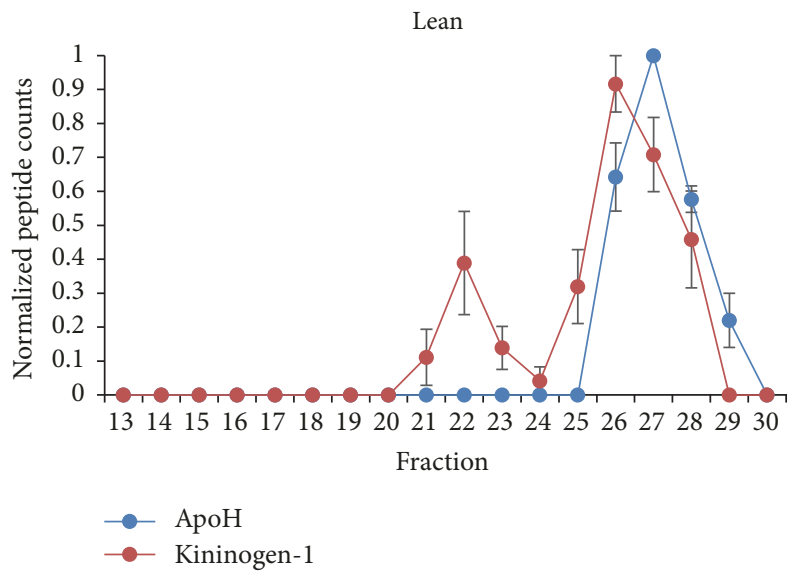

(e)

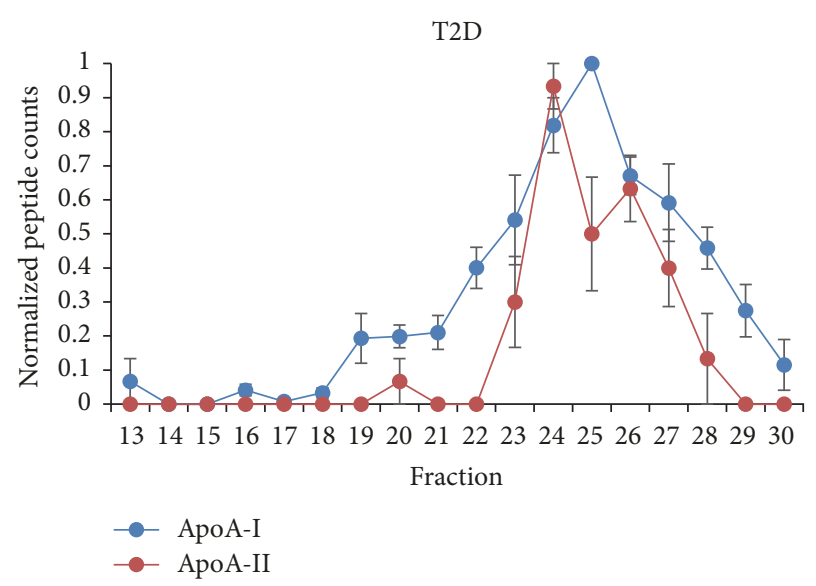

(b)

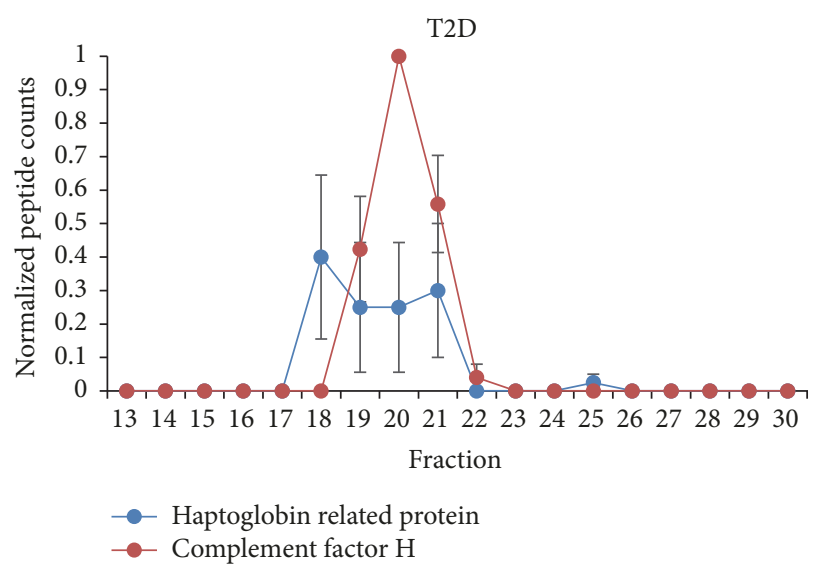

(d)

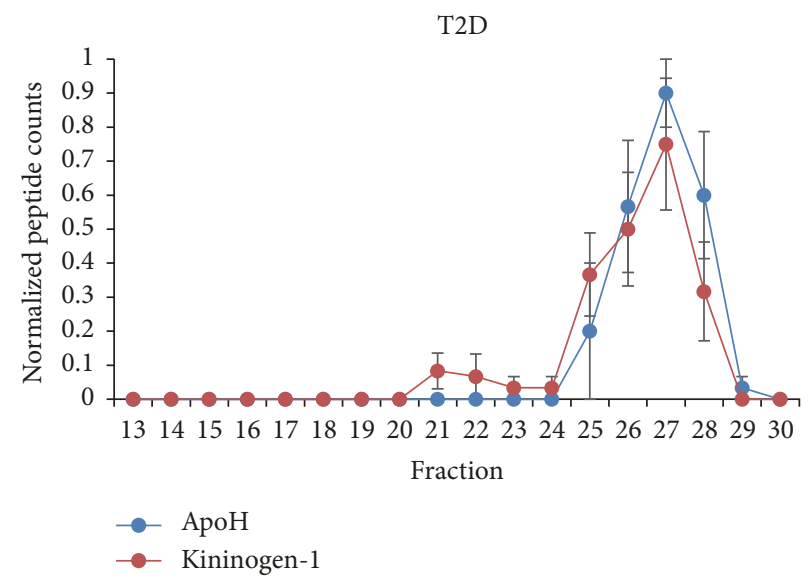

(f)

Figure 5: Comigration patterns of protein pairs. (a-b) apoA-I and apoA-II, (c-d) haptoglobin-related protein and complement factor H, and (e-f) apoH and kininogen-1, from lean and T2D groups. Spectral counts were normalized by dividing maximum value across all fractions. Data are mean and standard deviation of normalized spectral counts.

proatherogenic elements. Lastly, in the pink cluster (Figures 6(e) and 6(f)), four out of six members are positively PWVcorrelated proteins in T2D group. Only alpha-2-HS-glycoprotein demonstrates observable pattern change. Since these two clusters likely correspond to HDL subspecies in plasma, we expect these subspecies to be related to arterial stiffness.
3.4. HDL Subspecies and Disease. Taking our analysis a step further, we sought to explore the human diseases these subspecies are associated with. We used ToppCluster [31] to construct a disease-cluster association network $(P<0.05$ after Bonferroni correction). As shown in Figure 7, square nodes of the network represent all the diseases significantly 


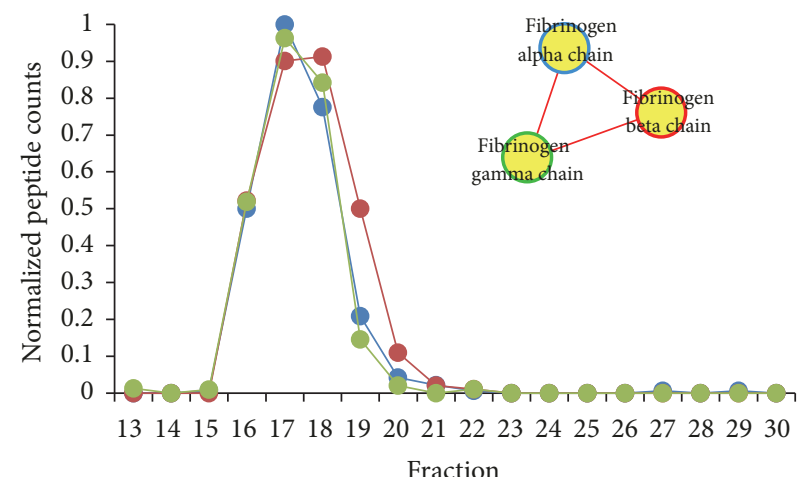

(a)

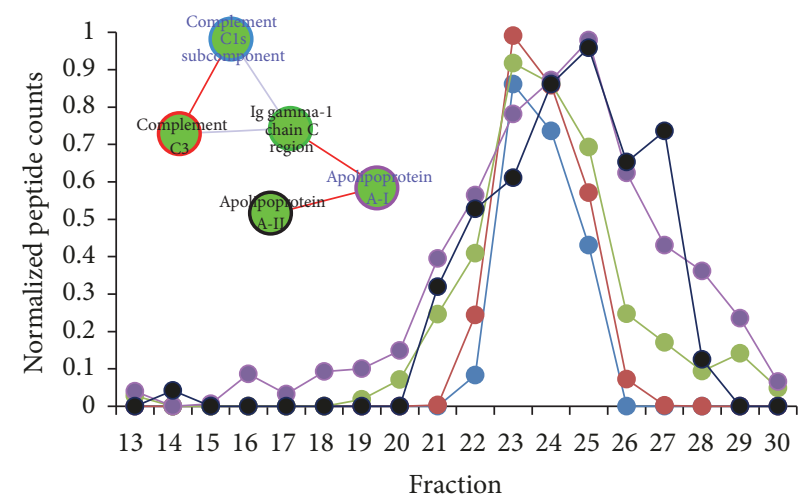

(c)

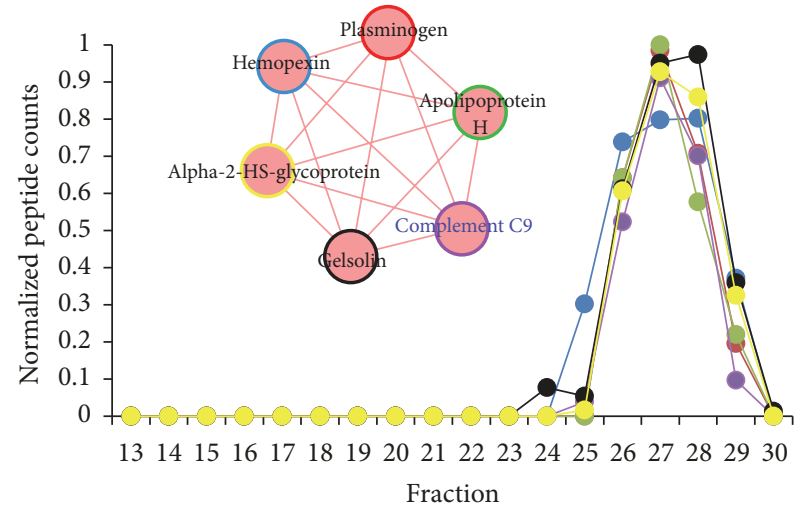

(e)

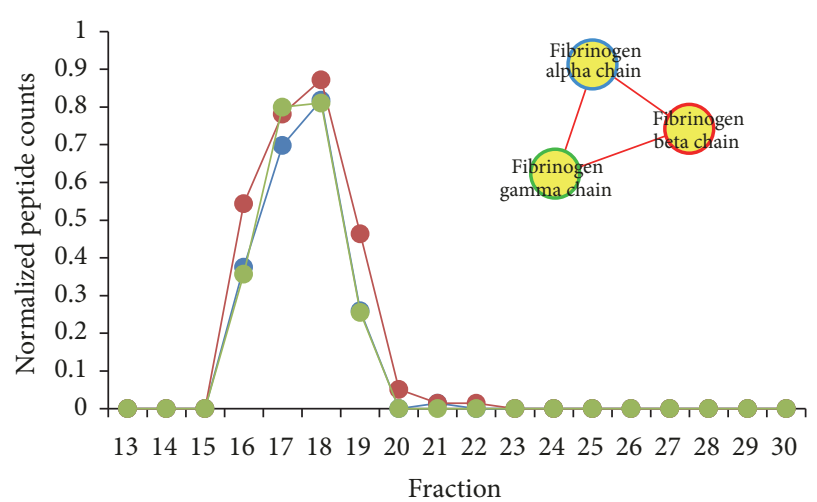

(b)

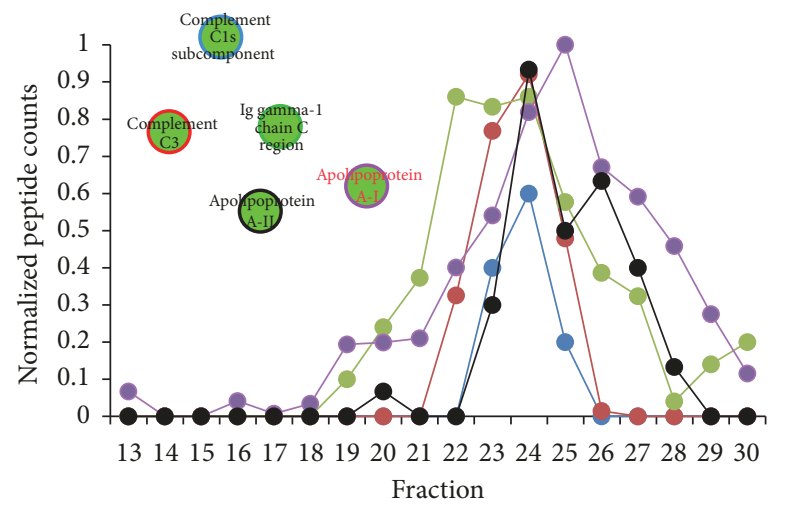

(d)

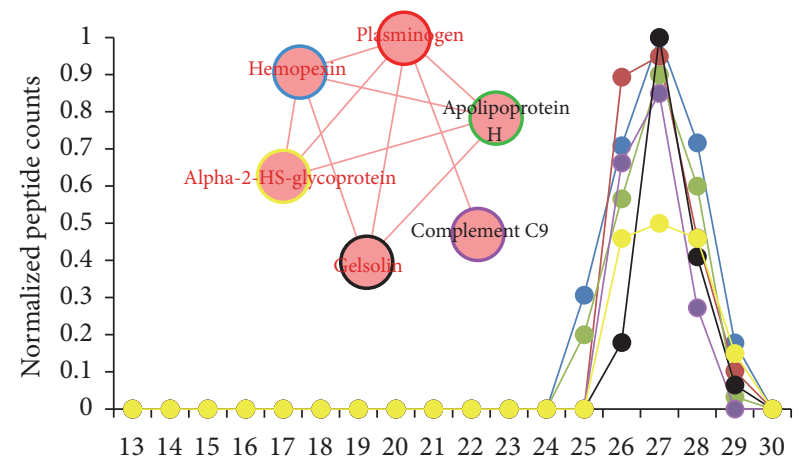

Fraction

(f)

FIGURE 6: Comigration patterns of the proteins residing on the same subspecies with at least two PWV-correlated proteins in the lean (a, $c$, e) and the T2D $(b, d, f)$ groups. Data are mean normalized peptide counts. Colors of distribution patterns are matched to the colors of node outlines. (a-b) Control: comigration pattern of fibrinogen $\alpha / \beta / Y$ chains (on the same particle) shows no difference between lean and T2D. (c-d) Cluster 1: apolipoprotein A-I, apolipoprotein A-II, Ig gamma-1 chain C region, complement C3, and complement C1s subcomponent. (e-f) Cluster 2: hemopexin, plasminogen, apolipoprotein $\mathrm{H}$, complement C9, gelsolin, and alpha-2-HS glycoprotein.

associated with those clusters. Edges in the network represent significant associations, which are calculated based on hypergeometric tests. We marked diabetes-related, CVD-related, and dyslipidemia-related terms with different colors. Both clusters appeared to be associated with certain CVD-related or dyslipidemia-related diseases. The strong associations indicate that the proteins within the subspecies may work together and contribute to the pathogenesis of those diseases.
Therefore, this disease association further supports that these HDL subspecies may be related to arterial stiffness.

\section{Discussion}

Many pieces of evidence have suggested that alterations of certain HDL proteins or subspecies in T2D patients may be associated with arterial stiffness [17, 33-37]. For example, 


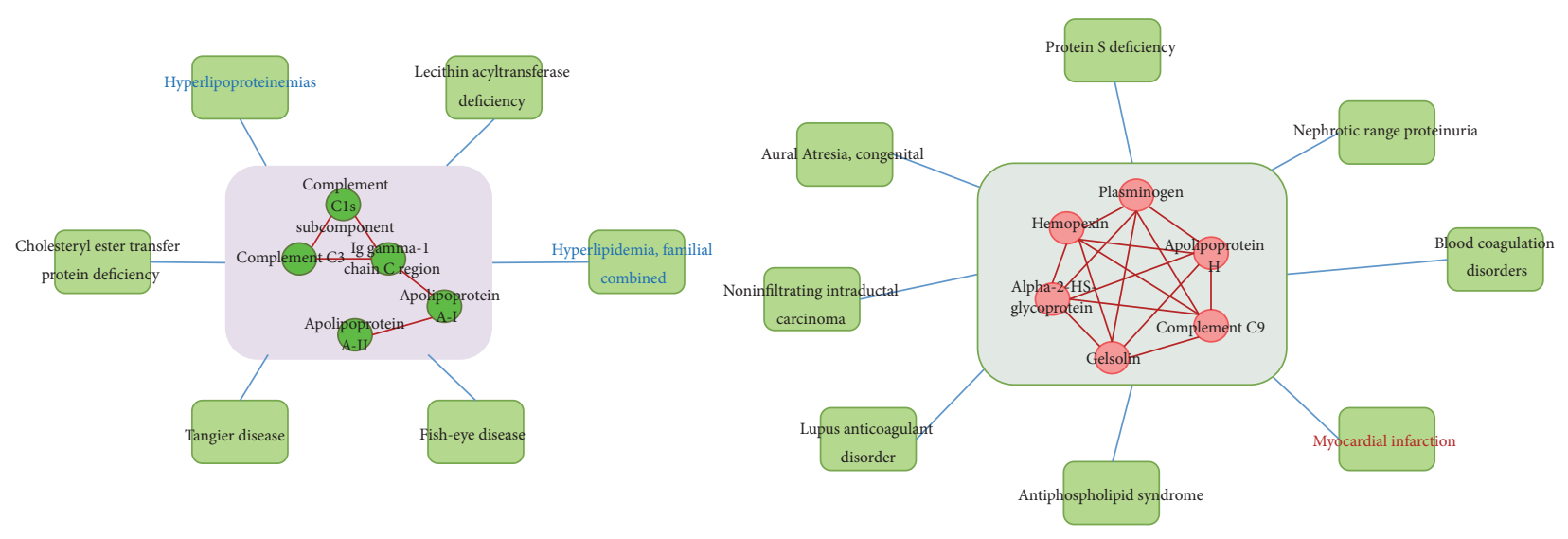

FIGURE 7: Disease-associated networks for two HDL subspecies. Each HDL subspecies is linked to its associated diseases (square nodes). Disease labels are marked with pink text (CVD-related), blue text (dyslipidemia-related), and black text (others).

endothelial protective activities of HDL isolated from T2D subjects are reduced compared to those of healthy control subjects [37]. Further, HDL incubated with glucose was shown to have impaired anti-inflammatory and antioxidant activities [36]. Thus, identification of T2D-specific HDL proteins or subspecies could not only serve as early biomarkers of distinct stages of CVD but also help in designing specific HDL-related therapies to protect persons with T2D from CVD complications.

Even though previous studies have shown that HDLassociated proteins are altered in youth with T2D [17], which proteins or subspecies are specifically related to arterial stiffness remains elusive. Here, we set out to identify HDL proteins and subspecies that correlate with arterial stiffness by comparing clinical and proteomics data from lean and T2D participants. This work is novel in two aspects: (1) it reveals a number of significantly PWV-correlated proteins by combining biological assays, clinical measurement, and HDL proteomics and (2) it goes beyond individual proteins and investigates the impact of T2D from a HDL subspecies perspective.

With regard to the individual HDL proteins that were correlated with arterial stiffness, several findings are noteworthy, including changes in the PCC of apoA-I, histidine-rich glycoprotein, and hemopexin with PWV. In the current analysis, apoA-I has both negative and positive correlations with arterial stiffness, which indicates that apoA-I may serve as a platform for HDL particles that can be both atheroprotective and atherogenic. Since the gel filtration technique separates HDL particles by hydrodynamic diameter, an alteration of the distribution pattern indicates a change in particle size and thus a compositional change of HDL subspecies. ApoA$\mathrm{I}$ in the T2D group exhibits lower normalized abundance in the larger HDL range and an increase in the smaller particle fractions. From the HDL subspecies perspective, apoA-I is the major component and structural scaffold of HDL and is likely involved in multiple HDL subspecies. Based on the PCC in different fractions (Figures 1 and 2), it is likely that the balance between larger size apoA-I containing subspecies in fractions 21-22 and the smaller apoA-I containing subspecies in fractions 25-28 may underlie apoA-I's apparent atheroprotection in the lean group and possibly also the increase in arterial stiffness in the T2D group. Unfortunately, based on current analysis, it is impossible to distinguish two possibilities: (1) T2D results in the breakdown of protective HDL particles into harmful smaller ones or (2) there exist two distinct HDL subspecies in the plasma with certain metabolic balance, and T2D tends to alter this balance. It will be important to understand the metabolic origins of these species to address these questions.

Additionally, histidine-rich glycoprotein and hemopexin are positively correlated with PWV in the T2D group. These two proteins play roles in the regulatory functions for the blood coagulation, complement, and fibrinolysis pathways. As of now, we do not have enough information to speculate how these proteins may contribute to vascular stiffness or even if they are causal in this regard.

Our network analysis revealed clear global differences in the protein networks between lean and T2D groups. Global topological parameters of these two networks suggest that the lean network has a stronger connectivity and tends to have more clusters, while the T2D network has a more scattered topology. It is possible that, in $\mathrm{T} 2 \mathrm{D}$, certain proteins are missing from critical HDL subspecies, thus altering their function. Fisher et al. [38] have described the formation of dysfunctional HDL. It is expected that when dysfunctional $\mathrm{HDL}$ are generated instead of mature spherical HDL, protein networks of HDL may show obvious topological differences. Our network analysis is consistent with the previous observations that the HDL subspecies in the T2D group are indeed altered by T2D [17, 33, 34], as demonstrated by the decreased intracluster links compared with the lean group (such as loss of the apoA-I and apoA-II link, as well as other links within the clusters).

Two candidate subspecies that contain at least two PWVcorrelated proteins that may be related to arterial stiffness were identified. Notably, there are some distribution pattern changes in individual proteins of the identified subspecies between the lean and T2D groups (Figure 6). This indicates that the composition of these particles may be altered in the T2D subjects. It is possible that such compositional 
alterations may impact certain functions of HDL and, consequently, the progression of CVD. These HDL subspecies may provide a link between $\mathrm{T} 2 \mathrm{D}$ and CVD complications. Currently, our study only focused on arterial stiffness and CVD. Since T2D has various typical complications (e.g., CVD, neuropathy, nephropathy, retinopathy, hearing impairment, and Alzheimer's disease), we speculate that diverse HDL subspecies or lipid-associated particles may be related to different complications.

One limitation of this work was the limited sample size. Due to the limitation of the subject recruiting as well as the relatively limited throughput of the plasma fractionation procedure, this study was carried out on a representative subject group instead of a large population. Thus, we cannot totally exclude the possibility of bias induced by individual differences. However, no other study has endeavored to subfractionate lipoproteins and track their proteome in this much detail. Another limitation of the study is that we cannot know with $100 \%$ certainty whether every protein in the analysis resided on an HDL particle. However, the study was specifically designed to increase the likelihood that we are only looking at HDL-associated proteins. The method used in this study involves a multipronged approach to fractionate HDL. First, whole plasma is size-fractionated. Second, LRA is used to isolate lipid-containing particles from each fraction. Although LRA effectively binds lipid-rich species, we cannot confidently exclude the possibility that some plasma proteins bound the LRA. Thus it is possible that, even with the LRA selection process, some plasma proteins were detected by MS analysis. However, finally, the resulting list of MS identified proteins (approximately 110 proteins) was filtered against the HDL Proteome Watch, a curated database that reports proteins that have been detected on HDL by multiple mass spectrometry studies. Thus, it is reasonable that the proteins reported in the final subspecies analysis are likely to be HDLassociated proteins. Future work will focus on validating these results in larger groups.

\section{Conclusions}

In summary, we identified seven HDL proteins that are negatively correlated with arterial stiffness, as well as nine proteins that positively correlated with arterial stiffness from complex proteomics data. Additionally, we constructed protein comigration networks for the lean and T2D groups, separately. Using the network-based complex identification, we discovered two PWV-related HDL subspecies that are associated with multiple complications of T2D. We caution that current findings are only based on correlation analysis. Nevertheless, the correlations are significant enough to support further experimental investigation. We found that the distribution of those proteins may provide a better understanding on how HDL proteins are altered in youth with T2D and how they are related to arterial stiffness. Our results also indicate that certain HDL proteins (e.g., apoA-I and histidine-rich glycoprotein) may reverse their protective roles and become atherogenic in $\mathrm{T} 2 \mathrm{D}$ condition. This may explain why the HDL-C raising therapies [7] have not achieved desirable outcomes. These PWV-related proteins and subspecies could be excellent targets in future deterministic experiments for investigating specific HDL subspecies.
Abbreviations
HDL: High-density lipoproteins
T2D: $\quad$ Type 2 diabetes
PWV: Pulse wave velocity
CVD: Cardiovascular disease
LDL: Low-density lipoproteins
HDL-C: High-density lipoprotein-cholesterol
TLF: Trypanosome lytic factor
apo: Apolipoprotein
RCT: Reverse cholesterol transport.

\section{Data Availability}

For data availability, please refer to the original study [17].

\section{Ethical Approval}

The study has been approved by IRB committee of Cincinnati Children's Hospital Medical Center.

\section{Consent}

Written informed consent was obtained from participants who are 18 years old or older or from the parent or guardian with for participants below 18 years of age.

\section{Conflicts of Interest}

The authors declare that there are no conflicts of interest.

\section{Authors' Contributions}

L. Jason Lu designed the research plan in this paper; Amy S. Shah and Scott M. Gordon performed experiments and collected clinical data; Xiaoting Zhu, Hailong Li, and Sheng Ren analyzed the data; Amy S. Shah, Debi K. Swertfeger, and John T. Melchior helped in study design; Xiaoting Zhu, Debi K. Swertfeger, Hailong Li, and L. Jason Lu wrote the manuscript; W. Sean Davidson and L. Jason Lu supervised the research.

\section{Acknowledgments}

This work was supported by National Institutes of Health (R01-HL111829 to L. Jason Lu, K23-HL118132 to Amy S. Shah, and R01-HL104136 to W. Sean Davidson), a predoctoral fellowship to Scott M. Gordon and postdoctoral fellowship to John T. Melchior from the Great Rivers Affiliate of the American Heart Association, and the National Natural Science Foundation of China (no. 31601083).

\section{Supplementary Materials}

Supplemental Figure 1: correlation of apoA-I relative abundance in fraction 21 versus PWV measurement. Lean, T2D, and combined data are marked with blue, red, and green, respectively. The PCC of combined data and PWV appear to be mainly driven by lean group, so that this PCC 
fails to reflect the relationship between PWV and proteins. (Supplementary Materials)

\section{References}

[1] S. M. Grundy and et al., "Prevention Conference VI: Diabetes and Cardiovascular Disease: Writing Group IV: Lifestyle and Medical Management of Risk Factors," Circulation, vol. 105, no. 18, pp. e153-e158, 2002.

[2] M.-R. Taskinen, "Diabetic dyslipidemia," Atherosclerosis Supplements, vol. 3, no. 1, pp. 47-51, 2002.

[3] J.-C. Fruchart, F. Sacks, M. P. Hermans et al., "The Residual Risk Reduction Initiative: A Call to Action to Reduce Residual Vascular Risk in Patients with Dyslipidemia," American Journal of Cardiology, vol. 102, no. 10, 2008.

[4] D. Keene, C. Price, M. J. Shun-Shin, and D. P. Francis, "Effect on cardiovascular risk of high density lipoprotein targeted drug treatments niacin, fibrates, and CETP inhibitors: meta-analysis of randomised controlled trials including 117,411 patients," British Medical Journal, vol. 349, Article ID g4379, 2014.

[5] R. S. Birjmohun, B. A. Hutten, J. J. P. Kastelein, and E. S. G. Stroes, "Efficacy and safety of high-density lipoprotein cholesterol-increasing compounds: A meta-analysis of randomized controlled trials," Journal of the American College of Cardiology, vol. 45, no. 2, pp. 185-197, 2005.

[6] S. J. Nicholls, H. B. Brewer, J. J. P. Kastelein et al., "Effects of the CETP inhibitor evacetrapib administered as monotherapy or in combination with statins on HDL and LDL cholesterol: a randomized controlled trial," Journal of the American Medical Association, vol. 306, no. 19, pp. 2099-2109, 2011.

[7] R. S. Wright, "Recent clinical trials evaluating benefit of drug therapy for modification of HDL cholesterol," Current Opinion in Cardiology, vol. 28, no. 4, pp. 389-398, 2013.

[8] L. Bowman, J. C. Hopewell, F. Chen et al., "Effects of anacetrapib in patients with atherosclerotic vascular disease," The New England Journal of Medicine, vol. 377, no. 13, pp. 1217-1227, 2017.

[9] F. Rezaee, B. Casetta, J. H. M. Levels, D. Speijer, and J. C. M. Meijers, "Proteomic analysis of high-density lipoprotein," Proteomics, vol. 6, no. 2, pp. 721-730, 2006.

[10] T. Vaisar, S. Pennathur, P. S. Green et al., "Shotgun proteomics implicates protease inhibition and complement activation in the antiinflammatory properties of HDL," The Journal of Clinical Investigation, vol. 117, no. 3, pp. 746-756, 2007.

[11] H. Karlsson, P. Leanderson, C. Tagesson, and M. Lindahl, "Lipoproteomics II: mapping of proteins in high-density lipoprotein using two-dimensional gel electrophoresis and mass spectrometry," Proteomics, vol. 5, no. 5, pp. 1431-1445, 2005.

[12] M. Heller, D. Stalder, E. Schlappritzi, G. Hayn, U. Matter, and A. Haeberli, "Mass spectrometry-based analytical tools for the molecular protein characterization of human plasma lipoproteins," Proteomics, vol. 5, no. 10, pp. 2619-2630, 2005.

[13] S. M. Gordon, J. Deng, L. J. Lu, and W. S. Davidson, "Proteomic characterization of human plasma high density lipoprotein fractionated by gel filtration chromatography," Journal of Proteome Research, vol. 9, no. 10, pp. 5239-5249, 2010.

[14] W. S. Davidson, R. A. G. D. Silva, S. Chantepie, W. R. Lagor, M. J. Chapman, and A. Kontush, "Proteomic analysis of defined hdl subpopulations reveals particle-specific protein clusters: relevance to antioxidative function," Arteriosclerosis, Thrombosis, and Vascular Biology, vol. 29, no. 6, pp. 870-876, 2009.
[15] J. Raper, R. Fung, J. Ghiso, V. Nussenzweig, and S. Tomlinson, "Characterization of a novel trypanosome lytic factor from human serum," Infection and Immunity, vol. 67, no. 4, pp. 19101916, 1999.

[16] H. Li, S. M. Gordon, X. Zhu et al., "Network-Based Analysis on Orthogonal Separation of Human Plasma Uncovers Distinct High Density Lipoprotein Complexes," Journal of Proteome Research, vol. 14, no. 8, pp. 3082-3094, 2015.

[17] S. M. Gordon, W. S. Davidson, E. M. Urbina et al., "The effects of type 2 diabetes on lipoprotein composition and arterial stiffness in male youth," Diabetes, vol. 62, no. 8, pp. 2958-2967, 2013.

[18] A. Heink, W. S. Davidson, D. K. Swertfeger, L. J. Lu, and A. S. Shah, "A Comparison of Methods to Enhance Protein Detection of Lipoproteins by Mass Spectrometry," Journal of Proteome Research, vol. 14, no. 7, pp. 2943-2950, 2015.

[19] S. M. Gordon, S. Hofmann, D. S. Askew, and W. S. Davidson, "High density lipoprotein: It's not just about lipid transport anymore," Trends in Endocrinology \& Metabolism, vol. 22, no. 1, pp. 9-15, 2011.

[20] J. A. Glomset, “The plasma lecithins: cholesterol acyltransferase reaction," Journal of Lipid Research, vol. 9, no. 2, pp. 155-167, 1968.

[21] M. I. Mackness, S. Arrol, and P. N. Durrington, "Paraoxonase prevents accumulation of lipoperoxides in low-density lipoprotein," FEBS Letters, vol. 286, no. 1-2, pp. 152-154, 1991.

[22] J.-R. Nofer and G. Assmann, "Atheroprotective effects of highdensity lipoprotein-associated lysosphingolipids," Trends in Cardiovascular Medicine, vol. 15, no. 7, pp. 265-271, 2005.

[23] C. Wadham, N. Albanese, J. Roberts et al., "High-density lipoproteins neutralize C-reactive protein proinflammatory activity," Circulation, vol. 109, no. 17, pp. 2116-2122, 2004.

[24] J. T. Kuvin, N. A. Harati, N. G. Pandian, R. M. Bojar, and K. R. Khabbaz, "Postoperative cardiac tamponade in the modern surgical era," The Annals of Thoracic Surgery, vol. 74, no. 4, pp. 1148-1153, 2002.

[25] S. Kulkarni, K. J. Woollard, S. Thomas, D. Oxley, and S. P. Jackson, "Conversion of platelets from a proaggregatory to a proinflammatory adhesive phenotype: Role of PAF in spatially regulating neutrophil adhesion and spreading," Blood, vol. 110, no. 6, pp. 1879-1886, 2007.

[26] American Diabetes Association, "Standards of medical care in diabetes-2012," Diabetes Care, vol. 35, pp. S11-S63, 2012.

[27] S. Laurent, J. Cockcroft, L. van Bortel et al., "Expert consensus document on arterial stiffness: methodological issues and clinical applications," European Heart Journal, vol. 27, no. 21, pp. 2588-2605, 2006.

[28] D. K. Swertfeger, H. Li, S. Rebholz et al., "Mapping atheroprotective functions and related proteins/lipoproteins in size fractionated human plasma," Molecular \& Cellular Proteomics, vol. 16, no. 4, pp. 680-693, 2017.

[29] W. S. Davidson, “HDL Proteome Watch, 2016," http://homepages.uc.edu/ davidswm/HDLproteome.html.

[30] T. Nepusz, H. Yu, and A. Paccanaro, "Detecting overlapping protein complexes in protein-protein interaction networks," Nature Methods, vol. 9, no. 5, pp. 471-472, 2012.

[31] V. Kaimal, E. E. Bardes, S. C. Tabar, A. G. Jegga, and B. J. Aronow, "ToppCluster: A multiple gene list feature analyzer for comparative enrichment clustering and networkbased dissection of biological systems," Nucleic Acids Research, vol. 38, no. 2, Article ID gkq418, pp. W96-W102, 2010. 
[32] S. M. Gordon and et al., "Multi-dimensional Co-separation Analysis Reveals ProteinProtein Interactions Defining Plasma Lipoprotein Subspecies," Molecular and Cellular Proteomics, vol. 12, no. 11, pp. 3123-3134, 2013.

[33] A. von Eckardstein and J.-R. Nofer, "Frail HDLs and stiff arteries in type 2 diabetes in juveniles," Diabetes, vol. 62 , no. 8, pp. $2662-$ 2664, 2013.

[34] J. W. Brinck, A. Thomas, E. Lauer et al., "Diabetes mellitus is associated with reduced high-density lipoprotein sphingosine1-phosphate content and impaired high-density lipoprotein cardiac cell protection," Arteriosclerosis, Thrombosis, and Vascular Biology, vol. 36, no. 5, pp. 817-824, 2016.

[35] C. Calvo, C. Talussot, G. Ponsin, and F. Berthézène, "Non enzymatic glycation of apolipoprotein A-I. Effects on its self-association and lipid binding properties," Biochemical and Biophysical Research Communications, vol. 153, no. 3, pp. 1060-1067, 1988.

[36] C. C. Hedrick, S. R. Thorpe, M.-X. Fu et al., "Glycation impairs high-density lipoprotein function," Diabetologia, vol. 43, no. 3 , pp. 312-320, 2000.

[37] S. A. Sorrentino, C. Besler, L. Rohrer et al., "Endothelial-vasoprotective effects of high-density lipoprotein are impaired in patients with type 2 diabetes mellitus but are improved after extended-release niacin therapy," Circulation, vol. 121, no. 1, pp. 110-122, 2010.

[38] E. A. Fisher, J. E. Feig, B. Hewing, S. L. Hazen, and J. D. Smith, "High-density lipoprotein function, dysfunction, and reverse cholesterol transport," Arteriosclerosis, Thrombosis, and Vascular Biology, vol. 32, no. 12, pp. 2813-2820, 2012. 


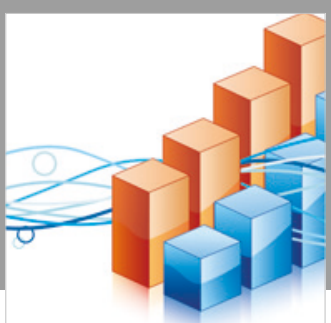

Advances in

Operations Research

\section{-n-m}
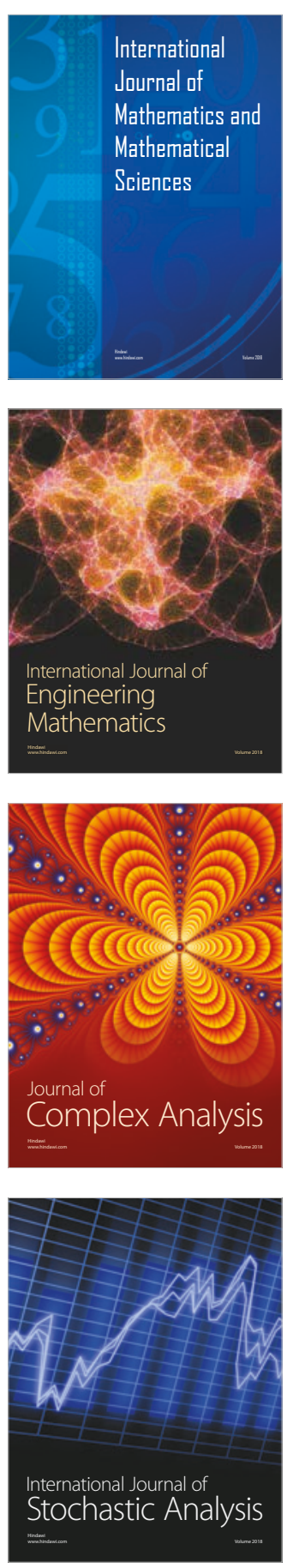
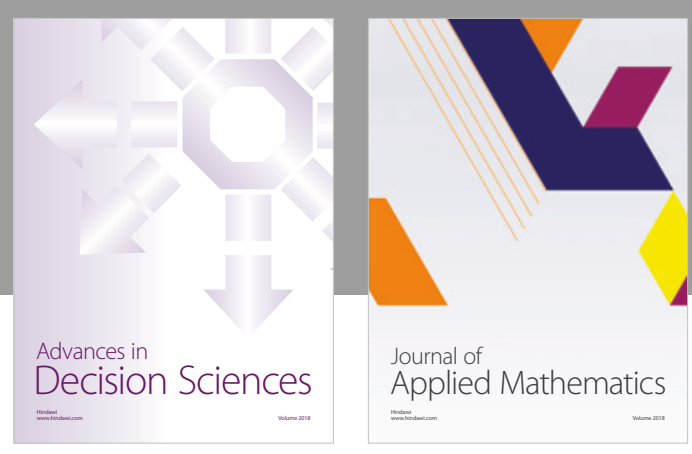

Journal of

Applied Mathematics
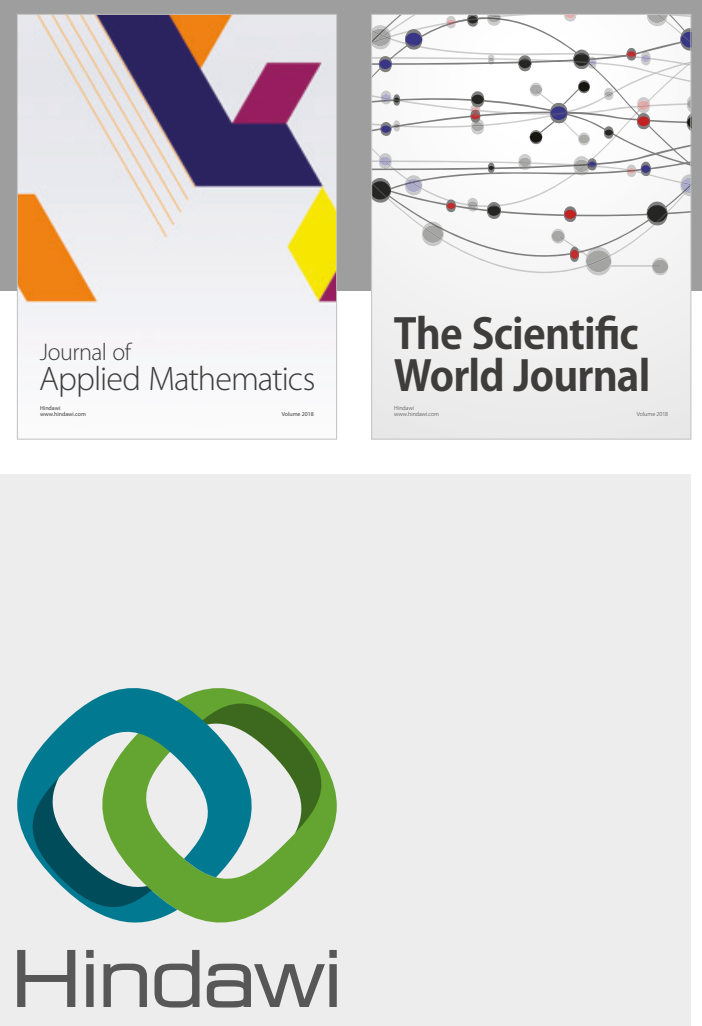

Submit your manuscripts at

www.hindawi.com

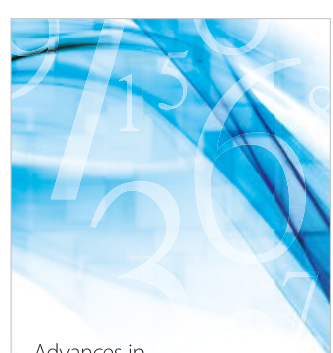

Advances in
Numerical Analysis
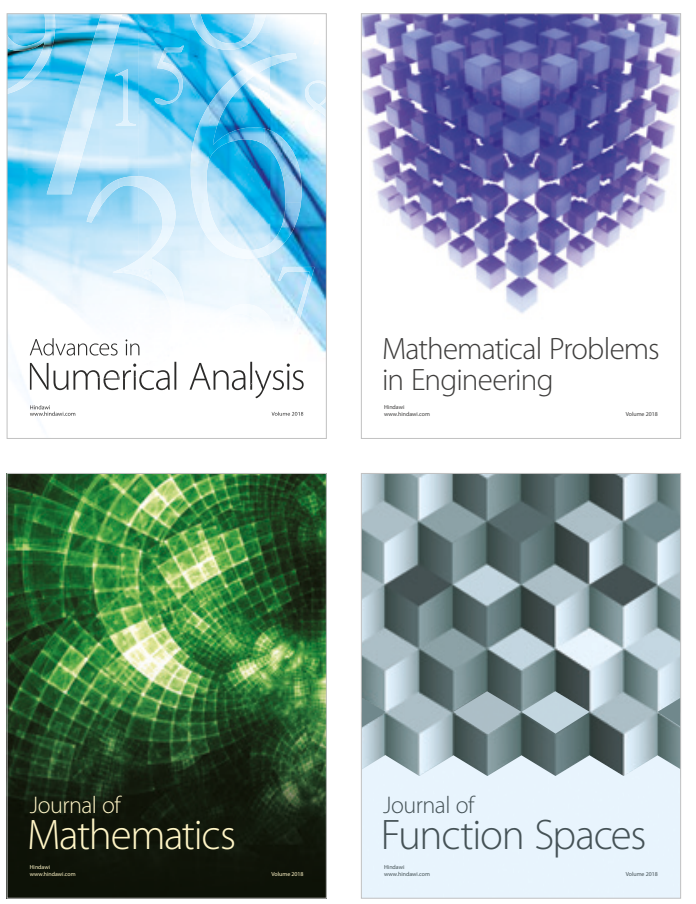

Mathematical Problems in Engineering

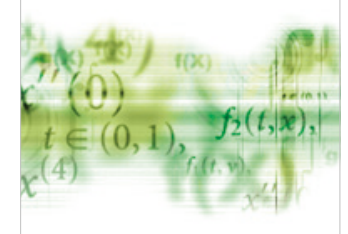

International Journal of

Differential Equations

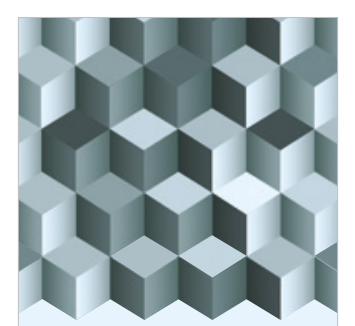

Journal of

Function Spaces
The Scientific

World Journal

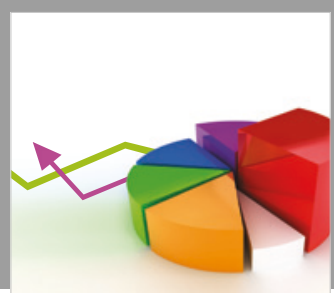

Journal of

Probability and Statistics
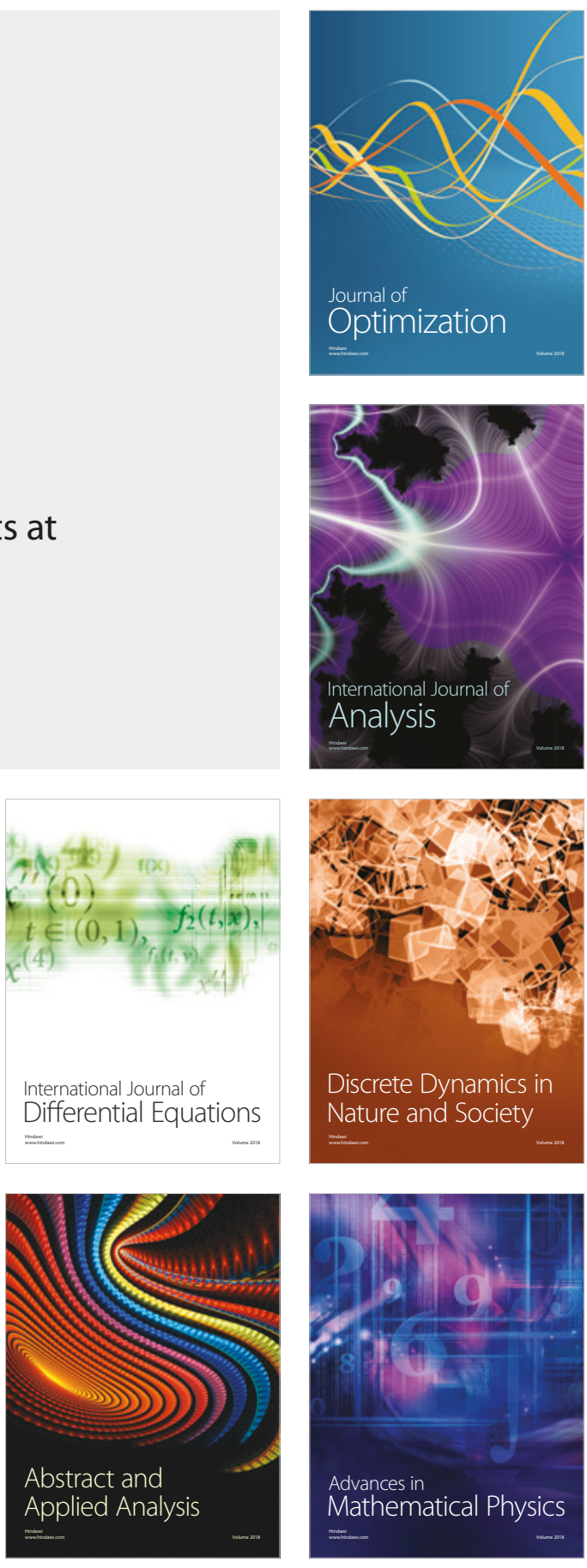